\title{
Lactobacillus rhamnosus GG: Experimental Evidence for a Clinical Utilization in Inflammatory Bowel Disease
}

\author{
Cristiano Pagnini* \\ "Sapienza" University of Rome, Faculty of Medicine and Psychology, S. Andrea Hospital, Rome, Italy
}

The last decades have seen a dramatic increase in the research of the intestinal microbiota, and, as a consequence, of the possible manipulation of the gut flora for therapeutic purposes by means of probiotic bacteria supplements. Accordingly, probiotic bacteria have been proposed for a plethora of pathologic situations, but real evidence for their application is limited to few diseases. In particular, probiotic utilization have been proposed in inflammatory bowel disease (IBD), that are two pathologic conditions (ulcerative colitis and Crohn's disease) of unknown etiology and immunologic pathogenesis, characterized by a chronic inflammation of the gut leading to a condition characterized by the alternation of flares and remission of disease. Despite studies on probiotic utilization in IBD have increased in the last years, to date the most solid evidence regards the use of probiotic in remission maintenance of pouchitis, while recent clinical data suggest a possible use in mild-moderate ulcerative colitis too [1]. Indeed, IBD represent a not homogenous condition that may present consistent differences in term of disease activity, location, course and behavior even inside the two distinct clinical entities, so that clinical studies, designed without appropriate selection of patients with specific disease features, may have led to disappointing results for the efficacy of probiotics. Moreover, probiotic studies examined many different formulations including mono- and multi-species compounds, each one with bacterial species with peculiar characteristics, with heterogeneous doses and treatment duration, so that results are not easily synthesizable [2,3].

Bearing in mind the aforementioned limits of the clinical studies, the most of the published studies about probiotics involves in vitro systems and experimental models of IBD [4]. Even though data from such studies are not directly reproducible in human condition, they may provide further insights into possible mechanism of action of probiotics and useful suggestion for specific clinical utilization of the bacteria in IBD patients. Among probiotc bacteria, Lactobacillus rhamnosus $G G$ (LGG) represents one of the more largely studied and it has demonstrated in vitro and in experimental model peculiar features suggesting a possible therapeutic utilization in IBD. In fact, considering together the recent advances in the field of microbiota research and in IBD pathogenesis, an ideal probiotic candidate for an efficacious usage in this setting should present three peculiar characteristics among the others: the capacity to adhere and persist at the intestinal mucosal level, to stimulate local innate immune response (i.e. epithelial functions), and to exert anti-inflammatory activity.

Although in vivo studies on mucosal adhesion of probiotic are scanty, in vitro experiments have shown that LGG presents an elevated binding capacity to the mucosal surface [5]. Genetic studies have recently shown the presence of pili that enhance the adhesive capacity of the bacteria [6]. Moreover, LGG can produce specific mucus binding proteins that further explain the high colonization capacity of this bacteria at mucosal level $[7,8]$. Further studies investigating the pattern of adhesion of LGG in the different segments of the colon might identify a subset of IBD patients with a localization of inflammatory disease that may be particularly eligible for therapeutic administration of LGG supplement. Similarly to other Lactobacilli, LGG electively interact with the intestinal epithelium with the result of a decrease of the intestinal permeability, stimulation of barrier effect through production of antibacterial molecules (i.e. mucins, defensins) and mucus, competition with pathogenic bacteria and local microflora modulation [9-11]. Most of the positive effect of the LGG on the epithelial function appears to be mediated by nuclear factor kB (NF-kB) modulation [12]. In addition, LGG may exert mucosal anti-inflammatory effect by a direct interaction with macrophages, dendritic cells and CD4+ lymphocytes, resulting in decreased production of pro-inflammatory cytokines, namely TNF, IL2, IL-4 [13-15].

In conclusion, even though solid evidence for a clinical effectiveness of probiotic bacteria in IBD are lacking, data from in vitro and experimental models indicate that LGG may represent a probiotic bacteria of particular interest for a clinical utilization in such conditions. Well-designed clinical studies are eagerly needed to properly translate into clinical setting the suggestion coming from the basic research. It is desirable that further evidences about the beneficial effects of probiotics and increased understanding of their underlying biochemical and immunological mechanisms of action will improve the treatment options for gut inflammation disorders and lead to a more rational and focused utilization of bacterial supplements in specific clinical conditions.

\section{References}

1. Meijer BJ, Dieleman LA (2011) Probiotics in the treatment of human inflammatory bowel diseases: update 2011. J Clin Gastroenterol 45 Suppl: S139-S144.

2. Mallon P, McKay D, Kirk S, Gardiner K (2007) Probiotics for induction of remission in ulcerative colitis. Cochrane Database Syst Rev: CD005573.

3. Butterworth AD, Thomas AG, Akobeng AK (2008) Probiotics for induction of remission in Crohn's disease. Cochrane Database Syst Rev: CD006634.

4. Claes IJ, De Keersmaecker SC, Vanderleyden J, Lebeer S (2011) Lessons from probiotic-host interaction studies in murine models of experimental colitis. Mol Nutr Food Res 55: 1441-1453.

5. Tuomola E, Crittenden R, Playne M, Isolauri E, Salminen S (2001) Quality assurance criteria for probiotic bacteria. Am J Clin Nutr 73: 393S-398S.

6. Kankainen M, Paulin L, Tynkkynen S, von Ossowski I, Reunanen J, et al (2009) Comparative genomic analysis of Lactobacillus rhamnosus GG reveals pili containing a human- mucus binding protein. Proc Natl Acad Sci U S A 106: 17193-17198.

7. von Ossowski I, Satokari R, Reunanen J, Lebeer S, De Keersmaecker SC et al. (2011) Functional characterization of a mucus-specific LPXTG surface

*Corresponding author: Cristiano Pagnini, M.D., Ph.D, "Sapienza" University of Rome, Faculty of Medicine and Psychology, S.Andrea Hospital, Via di Grottarossa 1035, 00189 Rome, Italy, E-mail: cristiano.pagnini@uniroma1.it

Received March 25, 2013; Accepted April 27, 2013; Published April 30, 2013

Citation: Pagnini C (2013) Lactobacillus rhamnosus GG: Experimental Evidence for a Clinical Utilization in Inflammatory Bowel Disease. Clin Microbial 2: 111 doi:10.4172/2327-5073.1000111

Copyright: (c) 2013 Pagnini C. This is an open-access article distributed under the terms of the Creative Commons Attribution License, which permits unrestricted use, distribution, and reproduction in any medium, provided the original author and source are credited. 
Citation: Pagnini C (2013) Lactobacillus rhamnosus GG: Experimental Evidence for a Clinical Utilization in Inflammatory Bowel Disease. Clin Microbial 2: 111. doi:10.4172/2327-5073.1000111

Page 2 of 2

adhesin from probiotic Lactobacillus rhamnosus GG. Appl Environ Microbiol 77: $4465-4472$

8. Vélez MP, Petrova MI, Lebeer S, Verhoeven TL, Claes I, et al. (2010) Characterization of MabA, a modulator of Lactobacillus rhamnosus GG adhesion and biofilm formation. FEMS Immunol Med Microbiol 59: 386-398.

9. Lebeer S, Vanderleyden J, De Keersmaecker SC (2008) Genes and molecules of lactobacilli supporting probiotic action. Microbiol Mol Biol Rev 72: 728-764, Table of Contents.

10. Wang Y, Liu Y, Sidhu A, Ma Z, McClain C, et al. (2012) Lactobacillus rhamnosus GG culture supernatant ameliorates acute alcohol-induced intestinal permeability and liver injury. Am J Physiol Gastrointest Liver Physiol 303: G3241.

11. Yan F, Cao H, Cover TL, Washington MK, Shi Y, et al. (2011) Colonspecific delivery of a probiotic-derived soluble protein ameliorates intestinal inflammation in mice through an EGFR-dependent mechanism. J Clin Invest 121: $2242-2253$
12. Donato KA, Gareau MG, Wang YJ, Sherman PM (2010) Lactobacillus rhamnosus GG attenuates interferon-\{gamma\} and tumour necrosis factoralpha-induced barrier dysfunction and pro-inflammatory signalling. Microbiology 156: 3288-3297.

13. Peña JA, Versalovic J (2003) Lactobacillus rhamnosus GG decreases TNFalpha production in lipopolysaccharide-activated murine macrophages by a contact-independent mechanism. Cell Microbiol 5: 277-285.

14. Braat $H$, van den Brande J, van Tol $E$, Hommes $D$, Peppelenbosch $M$, et al. (2004) Lactobacillus rhamnosus induces peripheral hyporesponsiveness in stimulated CD4+ T cells via modulation of dendritic cell function. Am J Clin Nutr 80: 1618-1625.

15. Donkor ON, Ravikumar M, Proudfoot O, Day SL, Apostolopoulos V, et al. (2012) Cytokine profile and induction of T helper type 17 and regulatory T cells by human peripheral mononuclear cells after microbial exposure. Clin Exp Immunol 167: 282-295. 